CERN-TH/95-139

\title{
CP-Violating Effect of QCD Vacuum in Quark Fragmentation
}

\author{
A. Efremov ${ }^{a, \text { f }}$ and D. Kharzeev ${ }^{b, c, \text { p }}$ \\ a) Laboratory of Theoretical Physics \\ Joint Institute for Nuclear Research \\ Dubna, 141980 Russia \\ $\left.{ }^{b}\right)$ Theory Division \\ CERN \\ Geneva, Switzerland \\ c) Fakultät für Physik \\ Universität Bielefeld \\ 33615 Bielefeld, Germany
}

\begin{abstract}
We demonstrate that the non-trivial CP-violating structure of $\mathrm{QCD}$ vacuum can lead to an observable effect in $e^{+} e^{-} \rightarrow q \bar{q} \rightarrow 2$-jet annihilation. We find that the sign of the jet handedness correlation can be opposite to that predicted by factorization of $q \bar{q}$ fragmentation and CP-conjugation of the two jets. A simple model estimation of the handedness correlation magnitude is given.
\end{abstract}

CERN-TH/95-139

May 1995

\footnotetext{
${ }^{1}$ E-mail: efremov@thsun1.jinr.dubna.su.

${ }^{2}$ E-mail: kharzeev@vxcern.cern.ch
} 
1. The problem of $\mathrm{CP}$ violation in strong interactions is of fundamental interest. Even though CP-violating effects are not expected in perturbative QCD, the nontrivial structure of QCD vacuum may lead to the breakdown of the invariance with respect to $\mathrm{CP}$ conjugation [1]. A particular illustration of this option is the appearance of the so-called $\Theta$-term in the effective Lagrangian of strong interactions:

$$
\mathrm{E}_{\Theta}=\frac{\Theta}{16 \pi^{2}} \operatorname{tr}\left(G_{\mu \nu} \tilde{G}^{\mu \nu}\right)
$$

which is intimately related to the possibility of tunnel transitions between the vacuum states with different topological indices [2]. This tunnelling phenomenon is invoked, for example, in the now generally accepted solution of the $U(1)$ problem in QCD [3]. It is easy to see that the term (1) explicitly breaks down CP invariance. However $\mathrm{CP}$-violation in strong interactions was never observed. Does it mean that the true theory of strong interactions is CP-invariant?

The answer to this question is not necessarily positive, since in the usual observables the non-perturbative CP-violating effects would appear only as tiny corrections, which are difficult to isolate. A possibility of CP-violation can be connected with solutions of classical equations of motion in gluodynamics with a nonzero field in the ground state [4]. It was suggested that such self-dual fields could provide color confinement and linearly rising Regge trajectories [5]. In fact, the existence of non-vanishing vacuum gluon fields assumed in the QCD Sum Rule method [6] would definitely induce the CP-violating effects due to a chromo-magnetic component of considerable strength:

$$
\left\langle\alpha_{s} B^{2}\right\rangle=\frac{1}{4}\left\langle\alpha_{s} G^{2}\right\rangle \simeq 0.01 \mathrm{GeV}^{4}
$$

where at a later stage we have used the "canonical" gluon condensate value [6]. However the terms induced by the vacuum gluon fields generally enter the expressions for physical quantities as higher-twist power corrections to the leading perturbative QCD results; these corrections are proportional to the square of the gluon field strength tensor $\left\langle\alpha_{s} G^{2}\right\rangle$. It is extremely important therefore to find an observable in which the non-perturbative effects would give a leading contribution.

Such an observable was recently proposed in the work of ref. [7]: it is the jet handedness correlation in the $e^{+} e^{-} \rightarrow Z^{0} \rightarrow 2$-jet process. There is a preliminary experimental indication [7] that this correlation is of the sign opposite to that predicted by CP-conjugation of $q$ and $\bar{q}$ jets and by their independent fragmentation.

The aim of this Letter is to demonstrate that the observed sign is natural if the fragmentation occurs in the background of non-perturbative chromo-magnetic vacuum field. We shall present a simple model estimation of the handedness correlation. 
2. Let us consider the process of $e^{+} e^{-}$annihilation into two jets:

$$
e^{+} e^{-} \rightarrow q+\bar{q} \rightarrow j e t_{q}+\text { jet }_{\bar{q}} .
$$

Since the $\bar{q} q$ pair is produced by the (spin 1) virtual photon or in the $Z^{0}$ decay, the spins of the quark and antiquark will be aligned in the same direction (opposite helicities). Consider now the pairs of positive and negative pions formed in the fragmentation of the jet. Let us call the pair "left" ("right") if the projection of the vector product of the three-momenta of positive and negative pions on the thrust vector $\vec{t}$ in the direction of the jet (i.e. the azimuthal angle $\phi$ of the pair with respect to the jet direction)

$$
\frac{\left(\vec{k}_{+} \times \vec{k}_{-}\right) \vec{t}}{\left|\vec{k}_{+}\right|\left|\vec{k}_{-}\right|}=\sin \phi
$$

is negative (positive). CP conjugation transforms a "left" ("right") pair produced in the quark-induced jet into the "left" ("right") pair in the antiquark-induced jet, and vice versa. The "handedness" [8] of the jet can be defined as

$$
H=\frac{N_{R}-N_{L}}{N_{L}+N_{R}},
$$

where $N_{L}\left(N_{R}\right)$ is the number of "left-handed" ("right-handed") pairs of charged pions formed in the fragmentation of the jet. This quantity is shown [8] to be proportional to the parent quark polarization $P_{q}$.

Let us now define also the "handedness correlation" parameter as

$$
C=\frac{N_{L R}+N_{R L}-N_{R R}-N_{L L}}{N_{L R}+N_{R L}+N_{R R}+N_{L L}},
$$

where $N_{L R}$, for example, stands for the number of events in which the pair in the quark-induced jet was "left" but the pair in the antiquark-induced jet was "right".

Naively, one would expect that the fragmentation of quark and antiquark jets is CP-symmetric, as it is in perturbative QCD. This means that a pair of some handedness in one jet has to correspond mainly to the pair of the same handedness in the opposite jet since the variable (46) is $\mathrm{CP}$-even, i.e. the correlation (6) has to be negative. This can be well illustrated in a simple classical model where the handedness arises from the turning of secondary quark and antiquark produced in breaking of string in a longitudinal chromo-magnetic field from chromo-magnetic dipole moments of initial $q$ and $\bar{q}$ [9]. Since the spin directions of initial $q$ and $\bar{q}$ are the same the directions of the chromo-magnetic fields are opposite. This results in the same handedness in both jets and in the negative correlation (6). There exists, however, an experimental indication [7] that it is definitely positive. This should mean that the $\mathrm{CP}$-conjugation of the two jets is broken. 
As we shall now discuss, an entirely non-perturbative component of the fragmentation - a vacuum gluon field, first considered in the context of jet physics in ref. [10] - could induce a local CP-parity violation and hence could lead to a positive value of $C$. Indeed, in the spirit of the model [9] the quark and antiquark "shaken-off" in the fragmentation process will be deviated by the background vacuum chromo-magnetic field (2) in the same direction, both in the quark- and antiquark-induced jets. The unit vector $\vec{n} \propto\left(\vec{k}_{+} \times \vec{k}_{-}\right)$will thus have a length different from zero and will be aligned in the same direction in both jets. This will induce an increase in the number of events with different "handedness" in opposite jets, $N_{L R}$ and $N_{R L}$, leading to the positive value of the "handedness correlation" (6).

3. Let us now present these qualitative arguments in a somewhat more formal way. The two-particle fragmentation function of a polarized quark fragmenting into a (+-)-pair in the background chromo-magnetic field $\vec{B}^{a}$ can be written down in the Lab system as

$$
D_{q}^{B}=w_{q}\left[1+\alpha_{q}(\vec{s} \vec{n})+\beta_{q}\left(\vec{B}^{a} \vec{n}\right)\right]
$$

where $\vec{s}$ is the spin of the quark, $w, \alpha$ and $\beta$ depend on the longitudinal and transverse (with respect to the thrust axis) momenta $k_{ \pm}^{L, T}$ of the particles and on the field strength $B^{2}$. Under the charge conjugation, $\vec{n}$ and $\vec{B}$ change sign, but $\vec{s}$ does not. Due to the charge conservation of fragmentation $D_{q}^{B}=D_{\bar{q}}^{-B}$ and

$$
\alpha_{\bar{q}}=-\alpha_{q}, \beta_{\bar{q}}=\beta_{q} .
$$

Averaging over different events with presumably random orientation of $\vec{B}$ and over azimuthal angle of $\vec{n}$, one obtains for handedness and handedness correlation in $e^{+} e^{-} \rightarrow$ 2-jet annihilation the following expressions (assuming naturally that $\langle\vec{B}\rangle=0$ ):

$$
H^{e^{+} e^{-}}=\frac{\sum_{q} \sigma_{q} w_{q} \alpha_{q} P_{q}}{\sum_{q} \sigma_{q} w_{q}} \text { and } C^{e^{+} e^{-}}=\frac{\Sigma_{q} \sigma_{q} w_{q}^{2}\left(-\alpha_{q}^{2} c_{q \bar{q}}+\beta_{q}^{2}\left\langle B^{2}\right\rangle\right)}{\Sigma_{q} \sigma_{q} w_{q}^{2}}
$$

where $P_{q}$ is the longitudinal quark polarization, $c_{q \bar{q}}=+1$ is the $q \bar{q}$ helicity correlation defined through the difference of the number of $q \bar{q}$ pairs with opposite helicities and with the same one and $\sigma_{q}$ is the production cross section of the flavour $q$. Formula (9) shows explicitly that the presence of a chromo-magnetic component of the gluon condensate of QCD vacuum can result in the positive sign of the handedness correlation (if the third term in (7) prevails) and hence to CP-asymmetry in the fragmentation of the two jets. Therefore, even though the parameter (6) is CP-even, its sign can indicate the presence of CP-asymmetry of the fragmentation process in QCD vacuum.

4. We shall now try to make some quantitative estimates. Assuming that the transverse size of the vacuum fluctuation $l$ and the transverse momentum $k_{T}$ of the "shaken-off" quark and antiquark satisfy the condition

$$
k_{T} l>>1
$$


one can estimate the magnitude of the effect in the classical approximation [10, 9]. Using (7), it is easy to see that the "handedness" (5) due to chromo-magnetic field $B$ is related to the angle $\chi$ of the deviation of quark (antiquark) from the back-to-back axis by the following relation, which is valid when the angle $\chi$ is small and one can use a linear (in $\sin \chi)$ approximation to the antisymmetric part of the distribution of the pair in the azimuthal angle $\phi$ :

$$
H_{B}=\langle\operatorname{sign}(\sin \phi)\rangle \simeq \frac{4}{\pi}\langle\sin \phi\rangle=\frac{4}{\pi}\langle\sin 2 \chi\rangle \simeq \frac{8}{\pi}\langle\chi\rangle
$$

where $\langle\ldots\rangle$ means averaging over the azimuthal angle. The "handedness correlation" parameter (6) in the presence of the field $B$ is given by

$$
C_{B} \simeq-(4 / \pi)^{2}\langle\sin \phi\rangle_{q-j e t}\left\langle\sin \phi^{\prime}\right\rangle_{\bar{q}-j e t}
$$

Since we consider the case of interaction with vacuum fields,

$$
\langle\sin \phi\rangle_{q-j e t}=-\left\langle\sin \phi^{\prime}\right\rangle_{\bar{q}-j e t}
$$

and we obtain

$$
C_{B} \simeq \frac{16}{\pi^{2}}\langle\sin \phi\rangle_{q-j e t}^{2} \simeq H_{B}^{2}
$$

Using the classical equation of motion of quark in the external chromo-magnetic field, we can write down the following expression for the mean deviation angle:

$$
\langle\chi\rangle \simeq \frac{\Delta k}{k_{T}} \simeq \frac{g B_{\|} \tau}{k_{T}}
$$

Here $\tau$ is the proper "formation time" of the meson 3 , i.e. the time during which the massless quark feels the chromo-magnetic field before it transforms to a color-neutral meson. In the jet center-of-mass system, it is given by [11]

$$
\tau \simeq \frac{1}{2 \epsilon_{\mathrm{c} . \mathrm{m} .}}=\frac{1}{2 k_{T} \cosh (y-Y)},
$$

where $\epsilon_{\mathrm{c} . \mathrm{m}}$. is the meson energy in the jet c.m. whereas $y$ and $Y=\frac{1}{2} \ln \left(\left(E_{j e t}-\right.\right.$ $\left.\left.P_{j e t}\right) /\left(E_{j e t}+P_{j e t}\right)\right)$ are the rapidities of the meson and of the center-of-mass of the jet in Lab system, respectively.

Turning to the correlation it is necessary to take into account that the fragmentation of $q$ and $\bar{q}$ takes place in different space-time points $x$ and $x^{\prime}$, and therefore the product of fields in different points will appear. These fields are not necessarily located in the same vacuum field domain. Averaging over all possible configurations of the domains restores the Lorentz invariance, making the product of the fields dependent on the interval $\left(x-x^{\prime}\right)^{2}$. The averaging also results in a suppression of the

\footnotetext{
${ }^{3}$ Recall that according to the uncertainty principle it is a minimal time during which a virtual fluctuation with energy deficit $\Delta E$ is undistinguishable from the initial state.
} 
bi-local product when the interval becomes larger than some correlation length $l$ (a "domain size") [5].

Recalling that for the longitudinal component of the chromo-magnetic field one has

$$
\left\langle g^{2} B_{\|}(x) B_{\|}\left(x^{\prime}\right)\right\rangle=\frac{1}{3}\left\langle g^{2} \vec{B}(x) \vec{B}\left(x^{\prime}\right)\right\rangle=\frac{\pi}{3}\left\langle\alpha_{s} G(x) G\left(x^{\prime}\right)\right\rangle,
$$

we obtain for the handedness correlation from (13), (11) the following expressionf:

$$
C \simeq \frac{64}{3 \pi}\left\langle\alpha_{s} G(x) G\left(x^{\prime}\right)\right\rangle \frac{\tau \tau^{\prime}}{k_{T} k_{T}^{\prime}},
$$

where the brackets $\langle\ldots\rangle$ denote averaging over all possible configurations of the domains.

The vacuum domain size is poorly known at present; to get an idea about the value of $l$ we can turn to the results of lattice calculations [12], which show that for large Euclidian space-time intervals $R^{2}=\left(t^{\prime}-t\right)^{2}+\left(\vec{r}-\vec{r}^{\prime}\right)^{2}$ the correlators $\left\langle G(x) G\left(x^{\prime}\right)\right\rangle$ fall off exponentially,

$$
\left\langle G(x) G\left(x^{\prime}\right)\right\rangle \simeq\left\langle G^{2}(0)\right\rangle \exp \left(-\frac{\sqrt{R^{2}}}{l}\right),
$$

with $l \simeq 0.2 \mathrm{fm}$. We will assume that this behavior holds for space-like intervals $\left(x^{\prime}-\right.$ $x)^{2}<0$ in Minkowski space-time since the change $t \rightarrow i t$ results in the substitution

$$
R^{2} \rightarrow-\left(t^{\prime}-t\right)^{2}+\left(\vec{r}^{\prime}-\vec{r}\right)^{2}=-\left(x^{\prime}-x\right)^{2} \simeq 4 t t^{\prime},
$$

where at the last stage we have taken into account that the points $x$ and $x^{\prime}$, over which the average is performed, are located in opposite fragmentation regions and quarks are moving approximately with the velocity of light. We thus get for space-like intervals

$$
C \simeq \frac{64}{3 \pi}\left\langle G^{2}(0)\right\rangle \exp \left(-2 \frac{\sqrt{t t^{\prime}}}{l}\right) \frac{\tau \tau^{\prime}}{k_{T} k_{T}^{\prime}} .
$$

Using for the gluon condensate the value (2) and assuming for the $k_{T}$ an average value $\left\langle k_{T}\right\rangle \simeq 0.3 \mathrm{GeV} / \mathrm{c}$ we find from (19) for the maximal value of the handedness correlation parameter at $\sqrt{t t^{\prime}} \simeq l \simeq 0.2 f m$ the value of the order of

$$
C \simeq \frac{64}{3 \pi}\left\langle G^{2}(0)\right\rangle e^{-2}\left(\frac{l}{\left\langle k_{T}\right\rangle \gamma}\right)^{2} \simeq+0.5 \%,
$$

where $\gamma=E_{j e t} / M_{j e t} \simeq 9$ is the Lorentz-factor for transformation from the Lab system to the jet center of mass system.

\footnotetext{
${ }^{4}$ We do not write down explicitly the Schwinger phase factor in the gluon field correlator required by gauge invariance.
} 
This value seems to underestimate the preliminary experimental observation [7]. However we have to stress once more that, first, we use a simple classical model which is hardly good for small $k_{T}$, second, the correlation length $l$ is poorly known (some estimations [5] give for it the value of up to $0.6 \mathrm{fm}$ ), and third, the observation [7] is very preliminary and selection of events made there is very severe. We would like to stress nevertheless in conclusion, that the positive value of $C$ corresponds to the CPviolating effect and this very non-trivial phenomenon deserves further experimental and theoretical investigation.

In particular, if it is really an effect of a vacuum field it should be accompanied also by an asymmetry corresponding to a vacuum chromo-electric field approximately of the same strength. It is not difficult to show that there has to be an asymmetry with respect to the difference of velocities of particles in pairs. Indeed, the covariant form of the third term in (可) is $G_{\mu \nu} k_{+}^{\mu} k_{-}^{\nu}$. In the product of the fragmentation functions for $q$ and $\bar{q}$ averaged over the vacuum field $G$ this results in a term

$$
\left\langle G G^{\prime}\right\rangle\left[\left(k_{+} k_{+}^{\prime}\right)\left(k_{-} k_{-}^{\prime}\right)-\left(k_{+} k_{-}^{\prime}\right)\left(k_{-} k_{+}^{\prime}\right)\right],
$$

because the only covariant and P-invariant form of the vacuum matrix element is

$$
\left\langle G_{\mu \nu}(x) G_{\mu^{\prime} \nu^{\prime}}\left(x^{\prime}\right)\right\rangle=\frac{1}{12}\left\langle G G^{\prime}\right\rangle\left(g_{\mu \mu^{\prime}} g_{\nu \nu^{\prime}}-g_{\mu \nu^{\prime}} g_{\nu \mu^{\prime}}\right) .
$$

The expression (21) can be rewritten as

$$
\left\langle G G^{\prime}\right\rangle \epsilon_{+} \epsilon_{+}^{\prime} \epsilon_{-} \epsilon_{-}^{\prime}\left[\left(\vec{v}_{+} \times \vec{v}_{-}\right)\left(\vec{v}_{+}^{\prime} \times \vec{v}_{-}^{\prime}\right)-\left(\vec{v}_{+}-\vec{v}_{-}\right)\left(\vec{v}_{+}^{\prime}-\vec{v}_{-}^{\prime}\right)\right],
$$

where $\vec{v}=\vec{k} / \epsilon$ is the velocity of a particle in a pair. While the first term in (22) is responsible for "chromo-magnetic" correlation, the longitudinal part of which has been considered above, the second one has to produce a "chromo-electric" correlation: the difference of velocity for particles in a pair in one jet should prefer to be directed in the opposite hemisphere to the difference in the opposite jet. It would be interesting to look for this effect experimentally.

The authors are grateful to J. Ellis, P. Minkowski, H. Satz, O. Teryaev, L.G. Tkatchev, V.I. Zakharov and to the referee of the first version of the paper for helpful discussions. A. E. acknowledges the hospitality of CERN Theory Division during the initial stage of this work. The work of A.E. was supported in part by the International Science Foundation under Grant FE300, by the INTAS Grant 93-1180 and by the Russian Foundation for Fundamental Research under Grant 93-02-3811. D. K. acknowledges financial support of the German Research Ministry (BMFT) under contract 06 BI 721 .

\section{References}

[1] See e.g. F. Wilczek, Phys. Rev. Lett. 40 (1978) 279. 
[2] A.A. Belavin, A.M. Polyakov, A.S. Schwartz and Yu.S. Tyupkin, Phys. Lett. B59 (1975) 85.

[3] G. 't Hooft, Phys. Rev. Lett. 37 (1976) 8;

G. Veneziano, Nucl. Phys. B159 (1979) 213;

E. Witten, Nucl. Phys. B156 (1979) 269;

D. I. Dyakonov and M. I. Eides, Sov. Phys. JETP 54 (1981) 232.

[4] G.K. Savvidi, Phys. Lett. B71 (1977) 133;

S.G. Matinyan and G.K.Savvidi, Nucl. Phys. B134 (1978) 539;

V.V. Skalozub, Sov. J. Nucl.Phys. 28 (1978) 113;

H. Pagels and E. Tomboulis, Nucl. Phys. B143 (1978) 485;

N.K. Nilsen and P. Olesen, Nucl. Phys. B144 (1978) 376;

P. Minkowski, Phys. Lett. B76 (1978) 439; Nucl. Phys. B177 (1981) 203;

H. Leutwyler, Nucl. Phys. B179 (1981) 129.

[5] H.G. Dosch and Yu.A. Simonov, Phys. Lett. B205 (1988) 339;

H.G. Dosch, Progr. Part. Nucl. Phys. 33 (1994) 121.

O. Nachtmann, Heidelberg University Preprint HD-THEP-94-42;

G. W. Botz, P. Haberl and O. Nachtmann, Z. Phys. C67 (1995) 143.

G.V. Efimov and S.N. Nedelko, Phys. Rev. D51 (1995) 176.

[6] M.A. Shifman, A.I. Vainshtein and V.I. Zakharov, Nucl. Phys. B147 (1979) $385 ; 448 ; 519$.

[7] A.V. Efremov, I.K. Potashnikova and L.G. Tkatchev, "Search for Jet Handedness Correlation in Hadronic Z-decays", presented at Rencontre de Moriond, Meribel, 1994. See also Proc. of 27th Int. Conf. on HEP, Glasgow, 1994, eds. Bussey P.J. and Knowles I.G., IOP, London, 1995, p. 875.

[8] A. Efremov, L. Mankiewicz and N. Törnqvist, Phys. Lett. B284 (1992) 394;

O. Nachtmann, Nucl. Phys. B127 (1977) 314;

A.V. Efremov, Sov. J. Nucl. Phys. 28 (1978) 83.

[9] M.G. Ryskin, Phys. Lett. B319 (1993) 346.

[10] M. Voloshin and V. Zakharov, Z. Phys. C6 (1980) 265.

[11] See e.g. B. Kopeliovich and F. Niedermayer, Yad. Fiz. 42 (1985) 797.

[12] A. Di Giacomo and H. Panagopoulos, Phys. Lett. B285 (1992) 133;

M. Campostrini, A. Di Giacomo and G. Mussardo, Z. Phys. C25 (1984) 173. 